\title{
Low-Cost Direct Electron Detection in the SEM for EBSD and ECCI
}

\author{
Joseph Tessmer ${ }^{1}$ and Marc De Graef ${ }^{2}$
}

${ }^{1}$ Carnegie Mellon University, PITTSBURGH, Pennsylvania, United States, ${ }^{2}$ Carnegie Mellon University, Pittsburgh, Pennsylvania, United States

SEM-based diffraction techniques are powerful tools for materials characterization. One of the most wellknown of these techniques is Electron Backscatter Diffraction (EBSD), which can be used to determine crystalline orientation near the surface of the sample. However, other diffraction techniques, such as the production of Electron Channeling Patterns (ECPs) can also be performed in the SEM. The orientation information obtained from the analysis of Electron Backscatter Patterns (EBSP) or ECP analysis can be used to perform Electron Channeling Contrast Imaging (ECCI). This technique involves orienting the sample such that only a single reflection is near Bragg orientation. This causes dislocations in the sample to appear clearly in Backscattered Electron (BSE) imaging. Images can be collected near several different Bragg orientations, allow the character of the dislocation to be identified. However, the most common commercial implementations of EBSD at this time involve purchasing a phosphor detector and software package which performs the work of collecting and indexing the EBSPs. This is fairly user-friendly, but can incur significant economic cost. This work implements a relatively low-cost direct electron detector in the SEM for use with collecting EBSPs. This can be combined with a dictionary-based indexing system such as that previously described in [1] to perform pattern collection and indexing.

This work makes use of a ADVACAM Minipix camera, which is designed primarily for x-ray detection. This detector is mounted inside the chamber of a TESCAN Mira-3 SEM with beam rocking capability, allowing ECPs to be collected in addition to EBSPs. The detector is currently mounted at a fixed angle, with plans to develop an adjustable mount that will enable detector angle and position to be varied more easily with respect to the sample to allow both EBSPs and ECPs to be collected using the same mount. The current mounting system lacks a cooling system, which means the detector cannot be continuously operated due to thermal concerns affecting the signal-to-noise ration of the detector. A more advanced mounting fixture will also include active cooling, which will allow the detector to operate indefinitely in the vacuum chamber.

There are several advantages to this detector configuration beyond economic incentives. Notably, the use of a pixelated direct electron detector allows for very fast collection of high-quality EBSPs com-pared to the commonly-used phosphor detectors (commercial direct electron detectors for EBSD are available, although at a higher cost than phosphor detectors.) The user also has direct control over the electron beam and detector hardware during scanning, allowing fine control over scan parameters. Additionally, the detector used here has energy-filtering capabilities. These can be used to isolate signal to specific electron energy bands. Comparison of this data to results predicted by different approaches to approximating the energy lost by electrons interacting with the sample (e.g. continuous slowing down approximation used in [2] or differential inverse inelastic mean free path [3].) Such results can be used to improve simulation algorithms for these diffraction modalities by providing more accurately information about the degree to which these energy bands each contribute to the overall pattern.

There are some drawbacks to this approach for EBSD when compared to a commercially available system. Significantly, there is no off-the-shelf mounting hardware that can be used to hold the detector in place in the vacuum chamber. This necessitates the design and construction of a mounting scheme by the user of the system. Additionally, while dictionary indexing can be used to index patterns once collected, it is necessary for the user to write a script or program that can externally control the electron beam and interface with the detector in order to quickly collect patterns in a grid. In this work, this control program was implemented through a python interface. This requires the user to write and test the collection software to ensure it is collecting patterns as intended. Of course, commercially available systems have software included which can 
be controlled through a GUI and performs pattern collection and analysis without requiring the user to interface with the electron beam control directly.

The system described in this work represents an opportunity for low-cost, high-speed access to orientation determine via the EBSD technique. There are also interesting potential applications for ECCI and model validation which make use of the energy-filtering capability of the detector. However, these advantages are tempered by the additional work required by the user to set up and operate the system. Figure 1 shows example EBSPs collected with different energy floors, and the EBSPs for energy bands between these floors obtained by image subtraction in postprocessing.
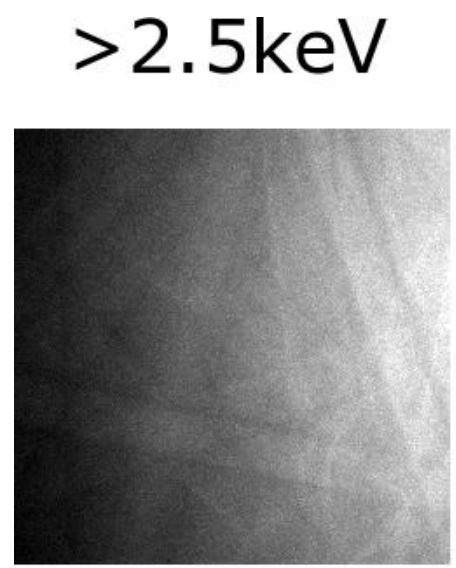
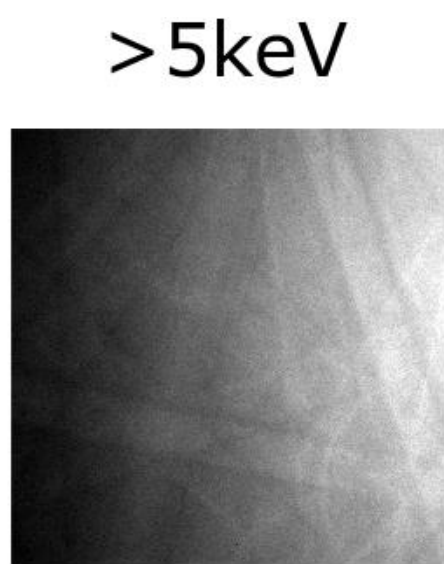

\section{$>10 \mathrm{keV}$}

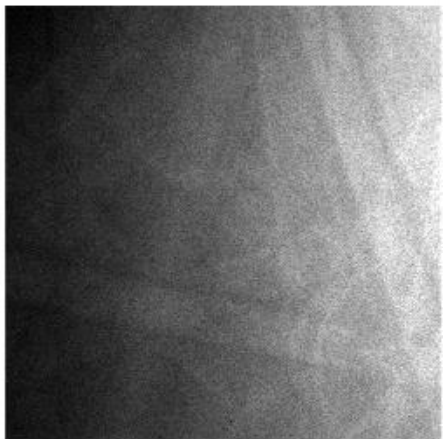

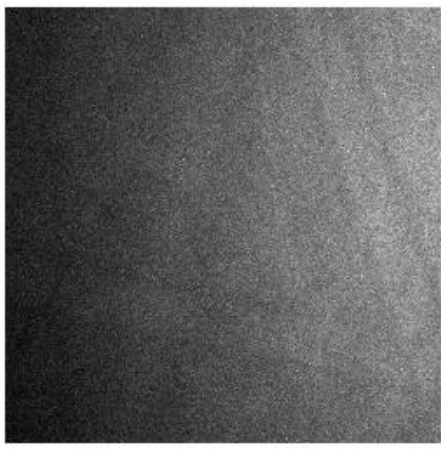

2.5-5keV

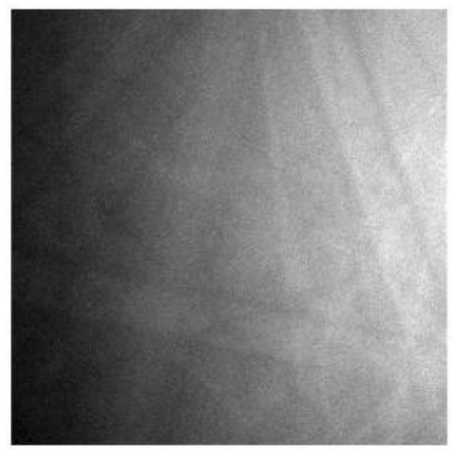

5-10keV

Figure 1. EBSPs collected from $\mathrm{Si}$ with a $15 \mathrm{kV}$ accelerating voltage. Each image label shows the electron energies which contributed to the pattern.

\section{References}

[1] Lenthe, W. C., S. Singh, and M. De Graef. "A spherical harmonic transform approach to the indexing of electron back-scattered diffraction patterns.’Ultramicroscopy 207 (2019): 112841.

[2] Callahan, Patrick G., and Marc De Graef. "Dynamical electron backscatter diffraction patterns. Part I: Pattern simulations. "Microscopy and Microanalysis 19.5 (2013): 1255.

[3] Salvat-Pujol, Francesc, and Wolfgang SM Werner. "Surface excitations in electron spectroscopy. Part I: dielectric formalism and Monte Carlo algorithm." Surface and interface analysis 45.5 (2013): 873-894.

[4] The authors acknowledge an ONR Vannevar Bush Faculty Fellowship (N00014-16-1-2821), and the computational resources of the Materials Characterization Facility at CMU, grant MCF-677785. 